\title{
Compressive radial nerve palsy induced by military shooting training: clinical and electrophysiological study
}

\author{
Woei-Cherng Shyu, Jiann-Chyun Lin, Ming-Key Chang, Wen-Long Tsao
}

\begin{abstract}
Ten recruited soldiers developed acute left wrist drop and numbness on the back of the thumb after a three hour military shooting training. Neurological examination disclosed decreased muscle power (0-2/5) of left wrist dorsiflexion, hypalgesia and hypaesthesia on the radial side of the left hand, and diminished brachioradialis reflex. Electrophysiological studies showed prolonged distal latency, reduced amplitude and slowness of left radial nerve motor conduction velocity between the axilla and elbow. Electromyography (EMG) revealed fibrillation potentials at rest, polyphasic motor unit and an incomplete interference pattern at volition over the extensor digitorum communis and brachioradialis. Nine patients recovered completely clinically and electrophysiologically between nine and 12 weeks after the onset of the palsy. Sensation recovered faster than the weakness. One patient failed to recover after three months, possibly because of the longer duration of nerve compression. Longer nerve compression time and sustained, decreased muscle power with signs of active denervation in EMG are indicators of poor prognosis.
\end{abstract}

(F Neurol Neurosurg Psychiatry 1993;56:890-893)

The radial nerve is frequently involved in either traumatic or non-traumatic injury. Severe radial nerve injury caused by a traumatic injury such as radial head fracture, operative complication, laceration wounds and elbow arthroscopy is usually associated with a poor prognosis. ${ }^{12}$ Non-traumatic radial injuries, such as crutch palsy, ${ }^{1}$ Saturday-night palsy, ${ }^{3}$ rheumatoid arthritis or fibrous band with radial nerve entrapment, ${ }^{45}$ wheel chair palsy, ${ }^{6}$ rifle-sling palsy, ${ }^{7-9}$ are transient and have a better prognosis. We reported that long-term compression due to persistent kneeling shooting posture caused a neurapraxia of the radial nerve. ${ }^{10}$ Ten such cases are presented to investigate the prognosis and recovery course of neurapraxic radial lesions by serial clinical and electrophysiological evaluations in human beings.

\section{Patients and methods}

Ten patients aged 20 years were treated in our outpatient department from August 1990 to July 1991. They all had a similar history of a sustained kneeling posture while shooting during a military training course. The entire course lasted for three hours interspersed with intermittent, three to five minute rest periods. Wrist drop developed between four and 24 hours after training. Normal blood biochemistry and radiograph examination excluded the possibilities of humeral fracture and systemic illness. Compressive radial neuropathy caused by the kneeling shooting posture was diagnosed by clinical and electrophysiological examinations, which were repeated weekly for about three months to evaluate the clinical course of the condition.

Dantac Neuromatric 2000 was used for nerve conduction and EMG studies. In reference to Young's report, ${ }^{11}$ we applied the standardised method to evaluate radial motor conduction, using a surface electrode for recording over the extensor digitorium communis. The site of distal stimulation was in the antecubital fossa at a point just lateral to the biceps tendon as it crosses the flexor crease. Proximal stimulation was given as high in the axilla as possible between the coracobrachialis and the long heads of the triceps. The recording electrode was placed $8 \mathrm{~cm}$ from the distal stimulation point over the extensor digitorium communis. This point was located at the junction of the upper and middle third of the forearm. In sensory conduction studies, a double ring surface electrode was placed over the major branch of the sensory nerve (superficial radial nerve) at the thumb. The ground was placed between the stimulation and recording sites. Antidromic stimulation was applied at the wrist $6.5 \mathrm{~cm}$ proximal to the recording electrode. The distance was measured with the wrist neutrally and thumb lightly adducted. The skin temperature of the forearm was kept at or above $34^{\circ} \mathrm{C}$ during electrophysiological examinations. Concentric needle electrodes were used for leading off from the brachial triceps, biceps, brachioradialis, extensor digitorium communis, first digitorium interosseus and abductor pollicis brevis.

\section{Results}

The radial nerve was compressed during the training course of military knee-arm kneeling shooting in 10 cases (fig 1). Other than case 4 , every patient underwent only one three hour session of kneeling shooting due to the development of wrist drop. Despite mildly weak wrist extension, case 4 underwent 
Figure 1 Shooting. posture and anatomical mechanism of radial nerve injury (inset).

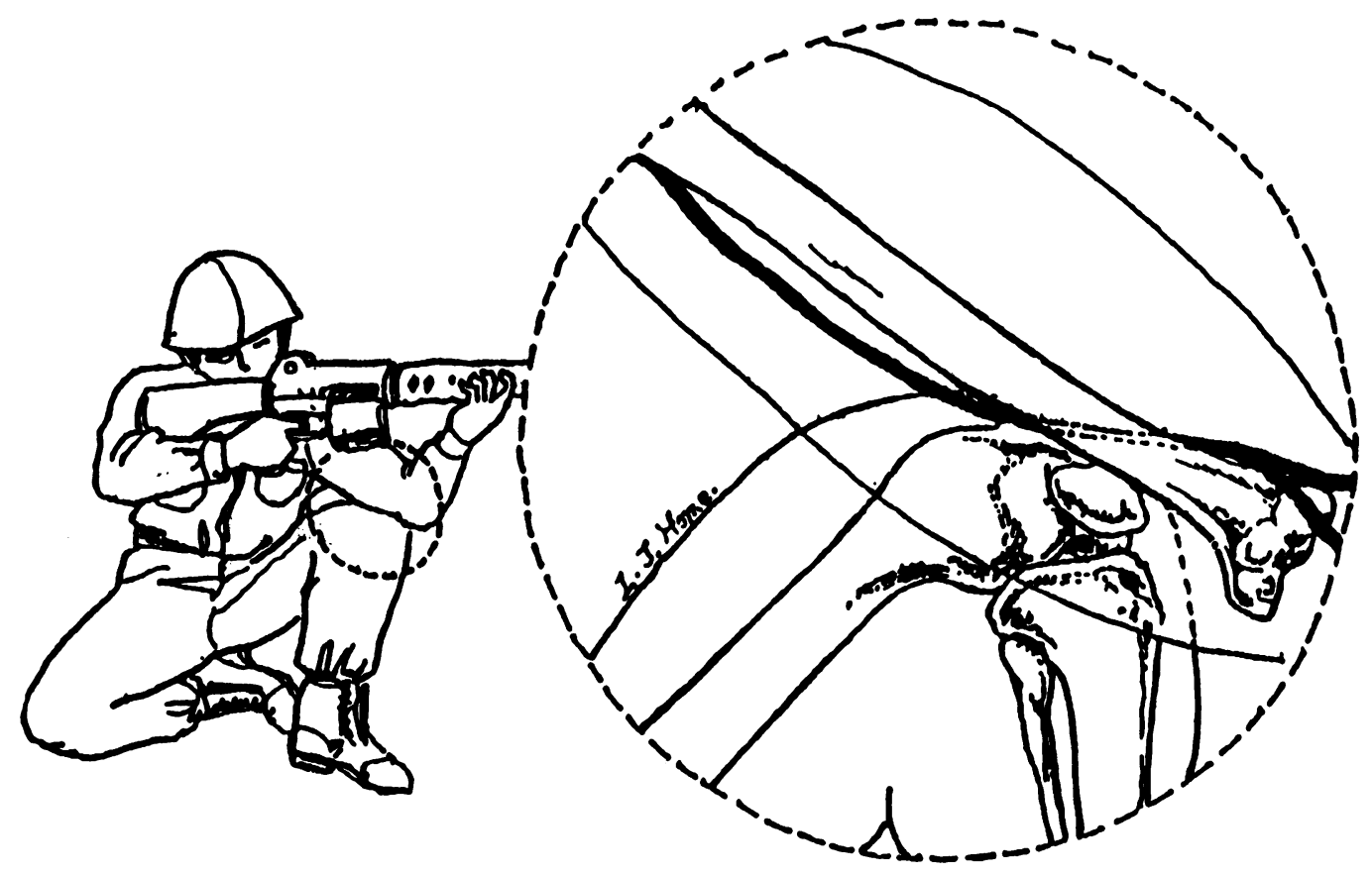

another three hour shooting training session on the second consecutive day.

When first investigated, the left brachioradialis and extensor of the wrist and fingers were affected in every patient, varying from complete paralysis (four cases) to moderate weakness (six cases). The left brachial triceps and left adductor muscles were spared in all patients. All showed diminished left brachioradialis deep tendon reflex. Nine out of 10 patients had hypaesthesia and hypalgesia over the dorsum of the thumb, index and middle fingers. One patient revealed sensory disturbances in the distribution of the forearm superficial radial nerve and posterior cutaneous nerve.

Radial nerve motor conduction studies showed prolonged distal latency and decreased conduction velocity between the axilla and elbow, that is, across the compressed segment of the nerve (table). There was a severe reduction in the motor response amplitudes evoked above the site of the lesion at first investigation which then gradually recovered, reaching normal values three to four weeks later. In sensory conduction studies, the amplitude and conduction velocity between the wrist and elbow were normal.

Table Initial motor conduction study of radial nerve and recovery time in patients with radial palsy induced by kneeling shooting training

\begin{tabular}{lccllc}
\hline $\begin{array}{l}\text { No. of } \\
\text { case }\end{array}$ & $\begin{array}{l}\text { Days from } \\
\text { injury }\end{array}$ & $\begin{array}{l}\text { DL } \\
(\mathrm{ms})\end{array}$ & $\begin{array}{l}\text { Amplitude } \\
(\mathrm{mV})\end{array}$ & $\begin{array}{l}\text { MNCV } \\
(\mathrm{m} / \mathrm{s})\end{array}$ & $\begin{array}{l}\text { Weeks to complete } \\
\text { recovery }\end{array}$ \\
\hline 1 & 6 & $4 \cdot 0$ & $0 \cdot 72$ & $38 \cdot 6$ & 11 \\
2 & 3 & $6 \cdot 0$ & $0 \cdot 85$ & $35 \cdot 5$ & 10 \\
3 & 5 & $5 \cdot 6$ & $0 \cdot 78$ & $38 \cdot 0$ & 9 \\
4 & 20 & $10 \cdot 0$ & $0 \cdot 44$ & $30 \cdot 0$ & $>12$ \\
5 & 5 & $3 \cdot 9$ & $1 \cdot 10$ & $44 \cdot 1$ & 9 \\
6 & 7 & $3 \cdot 8$ & 0.91 & $40 \cdot 6$ & 10 \\
7 & 5 & $4 \cdot 0$ & $0 \cdot 91$ & $41 \cdot 2$ & 10 \\
8 & 6 & $4 \cdot 3$ & $0 \cdot 94$ & $39 \cdot 4$ & 10 \\
9 & 7 & $3 \cdot 9$ & $0 \cdot 69$ & $38 \cdot 1$ & 12 \\
10 & 4 & $4 \cdot 1$ & $0 \cdot 88$ & $42 \cdot 1$ & 9 \\
\hline \multicolumn{7}{l}{ Normal (SD) } & $2 \cdot 7(0 \cdot 4)$ & $11 \cdot 0(3 \cdot 1)$ & $64 \cdot 0(7 \cdot 1)$ & \\
\hline
\end{tabular}

$\mathrm{DL}=$ distal latency; $\mathrm{MNCV}=$ motor nerve conduction velocity.
During EMG, the extensor digitorium communis and brachioradialis interference patterns were reduced at full effort in all 10 patients, and the amplitudes were below normal. Moreover, fibrillation potentials and polyphasic motor unit action potentials in the same muscles at full effort began to appear two weeks after the weakness became manifest.

Generally, patients began to recover three to four weeks after the nerve injury. The disappearance of sensory disturbances preceded that of wrist extension weakness. The time to recovery was 7.6 (SE 0.5) weeks (mean (SE), range five to 11 weeks) for sensory impairment and 10.0 (SE 0.3) weeks (range nine to 12 weeks) for weakness of wrist extension ( $p$ $<0.01)$. On the whole, the pattern of recovery was uniform for nearly all patients, health being regained nine to 12 weeks after the onset of symptoms (fig 2). The clinical observations, values for radial nerve motor conduction velocities between the axilla and elbow and EMG interference patterns in case 2 were plotted as a function of time (fig 3 ).

Unfortunately, one patient had not recovered after the three month follow up. There was still a reduction of the motor response amplitude and slow motor conduction velocity $(49 \mathrm{~m} / \mathrm{s})$ at the last examination. Clinically, muscle power was only grade 3 and sensory impairment persisted over the dorsum of the thumb, index and middle fingers.

\section{Discussion}

The clinical manifestations varied in patients with radial nerve compression sustained during military kneeling shooting training. The lesion in this radial palsy is located at the lateral border of the humerus, where the radial nerve pierces the lateral intermuscular septum; here the nerve is superficial and close to the humerus. Compression over this point causes paresis of the brachioradialis and 


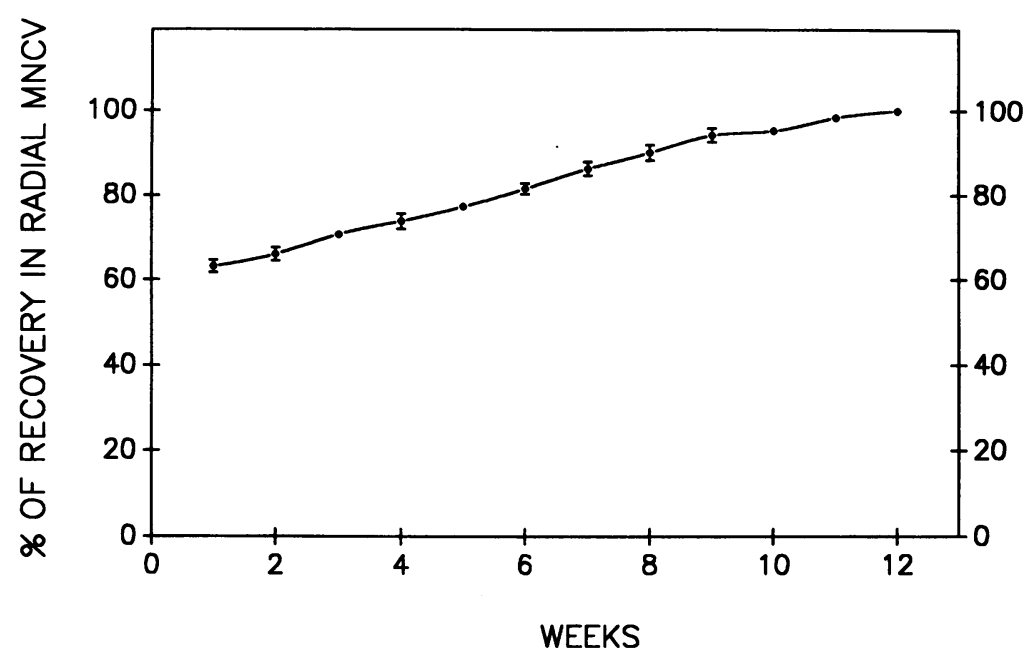

Figure 2 Recovery course of radial motor nerve conduction velocity (MNCV) in nine patients. Data expressed as mean (SE). In the five points without bars, $S E$ was one-half of the symbol or less. it should obviously be normal between the wrist and elbow. We suspect that abnormal sensory conduction velocity would have been disclosed if stimulation had been given between elbow and axilla. ${ }^{12}$

If nerve compression continues over two hours, nerve palsy will develop. ${ }^{13}$ The origin of the posterior cutaneous nerve of the forearm from the main trunk of the radial nerve varies from 8 to $30 \mathrm{~cm}$ with reference to the acromion. ${ }^{14}$ The vulnerability of the posterior cutaneous branch of radial nerve to compression may depend on anatomical variations. The organisation of sensory fibres in the anterior, central and medial positions of the radial nerve explains the less pronounced sensory disorder and the faster recovery of sensation. ${ }^{15}$ Furthermore, sensory fibres are less susceptible to pressure than motor fibres in both humans and animals. ${ }^{16}$

According to the Seddon classification, the pattern of this nerve injury is neurapraxia. It was confirmed that the radial nerve conduction velocity was slow, from our and other reports. ${ }^{17}$ In Spencer and Spenier's report, ${ }^{18}$ nerve injuries are classified as mild brief compression, moderate compression, slow progressive chronic compression, severe transient compression and severe chronic compression. Referring to the clinical symptoms and course of recovery, our cases coincided with the moderate compression category. In this type of lesion, there may be local conduction block which does not recover until several weeks after the compression is released. The recovery time of dissimilar patterns of radial nerve palsy ranged from three weeks to three months. ${ }^{61219}$ It may be due to difference of pressure sites, duration of compression, race and even environment.

In moderate nerve compression, histopathological studies indicate local demyelination of the large myelinated fibres with an intact axon, especially at the proximal edge of the compression site. ${ }^{1820}$ In other words, recovery is dependent on remyelination of locally denuded areas of axon, a process that continues over a period of several weeks. One of our patients had not recovered completely three months after the onset. We assume that it might be related to the longer duration of his radial nerve compression. Epineurial fibrosis with adhesions induced by long term compression, or even axonal damage with Wallerian degeneration would have a poor prognosis. ${ }^{1221}$ Neurolysis or tendon transfer to achieve functional recovery might be helpful for these cases. ${ }^{22}$

There are two mechanisms involved in this type of radial palsy. The first major mechanism might be lack of epineurial tissue at the site where the radial nerve passes the spiral groove of the humerus, thereby losing support and protection. ${ }^{1}$ Direct compression as the nerve passes the lateral tendon of triceps during strenuous exercise is the other possible cause. $^{23}$

The differential diagnosis includes hereditary pressure-sensitive neuropathy ${ }^{24}$ and an underlying polyneuropathy. Negative history 
of previous compressive nerve palsies and normal conduction velocities of the median, ulnar, peroneal, tibial and sural nerves excluded these two possible entities in our patients.

1 Sunderland S. Nerve and nerve injury. 2nd edn. Edinburgh: Churchill Livingstone, 1978:65-71.

2 Thomas MA, Fast A, Shapora D. Radial nerve damage as a complication of elbow arthroscopy. Clin Orthop 1987; 215:130-1.

3 Gernandez E, Pallini R, Talamonti G. Sleep palsy (Saturday-night palsy) of the deep radial nerve-case report. F Neurosurg 1987;66:460-1.

4 Dyck PJ. Peripheral neuropathy. 2nd edn. Philadelphia: WB Saunders: 1984:114-6.

5 Nielsen HO. Posterior interosseous nerve paralysis caused by fibrous band compression at supinator muscle. Acta Orthop Scand 1976;47:304-7.

6 Hartigan JD, Connolly TJ. Is 'wheel-chair wrist palsy' a new syndrome to watch for? Geriatrics 1990;45:63-71.

7 Burke EL, Glenn CG, Wales JF. Rifle-sling palsy in marine corps recruits. U.S. Armed Forces Med $\mathcal{F} 1957$; 8:1189-94.

8 Muntz HH. Connrad RW, Murchison RA. Rifle-sling palsy. U.S. Armed Forces Med f 1955;6:353-7.

9 Weinstein EA. Localized non-traumatic neuropathy in military personnel. Arch Neurol Psychiatry 1947;57: 369-76

10 Shyu WC, Shean HS, Hwang TY, Lan SJ. Compressive radial nerve palsy induced by military shooting training-a case report. Kaohsiung $f$ Med Sci 1991;7:334-7.

11 Young AW, Redmond MD, Memler DE, Belanders PV. Radial motor nerve conduction studies. Arch Phys Med
Rehabil 1990;71:399-402.

12 Trojabrog $\mathrm{W}$. Rate of recovery in motor and sensory fibers of the radial nerve: clinical and EMG study. $f$ Neurol Neurosurg Psychiatry 1970;33:625-38.

13 Lewis T, Pockering GW, Rothschild P. Centripedal paralysis arising out of arrested blood flow to the limb, including notes on a form of tingling. Heart Lung 1963; including

14 Linell EA. The distribution of nerve in the upper limb with reference to variability and their clinical significance. F Anat 1956;55:79-112.

15 Sunderland S. Traumatic injuries of peripheral nerve. I simple compression injuries of the radial nerve. Brain 1945;68:56-72

16 Mayer RF, Denny-Brown D. Conduction velocity in peripheral nerve during experimental demylination in the cat. Neurology 1964;14:714-26.

17 Downie AW, Scott TR. Radial nerve conduction studies. Neurology 1964;14:839-43.

18 Spencer M, Spenier PS. Nerve compression lesions of the upper extremity - a clinical and experimental review. upper extremity - a clinical

19 Preston DM, Grimes JD. Radial compression neuropathy in advanced Parkinson's disease. Arch Neurol 1985;42: 695-6.

20 Denny-Brown D, Brenner C. Paralysis of nerve induced by direct pressure and by tourniquet. Arch Neurol Psychiatry 1944;51:1-26.

21 Burns J, Lister GD. Localized constrictive radial neuropathy in the absence of extrinsic compression-three cases. $\mathcal{F}$ Hand Surg 1984;9:99-103.

22 Reid RL. Radial nerve palsy. Hand Clin 1988;4:179-85.

23 Mitsunage MM, Nsknso $\mathrm{K}$. High radial nerve palsy following strenuous muscle activity-a case report. Clin Orthop 1988;234:39-42.

24 Bense F, Buchthal F, Carlsen F. Hereditary neuropathy with liability to pressure palsies. Electrophysiological and histopathological aspects. Brain 1972;95:777-94. 